\title{
Meta and Media Data Stream Forensics in the Encrypted Domain of Video Conferences
}

\author{
Robert Altschaffel \\ Jonas Hielscher \\ Stefan Kiltz \\ Jana Dittmann \\ robert.altschaffel@iti.cs.uni-magdeburg.de \\ jonas.hielscher@iti.cs.uni-magdeburg.de \\ kiltz@iti.cs.uni-magdeburg.de \\ jana.dittmann@iti.cs.uni-magdeburg.de \\ Research Group Multimedia and Security \\ Otto-von-Guericke University \\ Magdeburg, Germany
}

\begin{abstract}
Our paper presents a systematic approach to investigate whether and how events can be identified and extracted during the use of video conferencing software. Our approach is based on the encrypted meta and multimedia data exchanged during video conference sessions. It relies on the network data stream which contains data interpretable without decryption (plain data) and encrypted data (encrypted content) some of which is decrypted using our approach (decrypted content). This systematic approach uses a forensic process model and the fission of network data streams before applying methods on the specific individual data types. Our approach is applied exemplary to the Zoom Videoconferencing Service $\left(V C S_{A}\right)$ with Client Version 5.4.57862.0110 [4], the mobile Android App Client Version 5.5.2 (1328) [4], the webbased client and the servers (accessed between Jan 21st and Feb 4th). The investigation includes over 50 different configurations. For the heuristic speaker identification, two series of nine sets for eight different speakers are collected. The results show that various user data can be derived from characteristics of $V C S_{A}$ encrypted media streams, even if end-to-end encryption is used. The findings suggest user privacy risks. Our approach offers the identification of various events, which enable activity tracking (e.g. camera on/off, increased activity in front of camera) by evaluating heuristic features of the network streams. Further research into user identification within the encrypted audio stream based on pattern recognition using heuristic features of the corresponding network data stream is conducted and suggests the possibility to identify users within a specific set.
\end{abstract}

\section{CCS Concepts}

- Applied computing $\rightarrow$ Computer forensics.

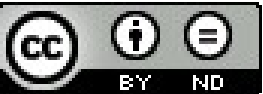

This work is licensed under a Creative Commons Attribution-NoDerivs International 4.0 License..

IH\&MMSec '21, fune 22-25, 2021, Virtual Event, Belgium.

(C) 2021 Copyright held by the owner/author(s).

ACM ISBN 978-1-4503-8295-3/21/06.

https://doi.org/10.1145/3437880.3460412

\section{Keywords}

User identification, Media Stream Forensics, Network Forensics, Meta Data Privacy, Video Conferencing

ACM Reference Format:

Robert Altschaffel, Jonas Hielscher, Stefan Kiltz, and Jana Dittmann. 2021. Meta and Media Data Stream Forensics in the Encrypted Domain of Video Conferences. In Proceedings of the 2021 ACM Workshop on Information Hiding and Multimedia Security (IH\&MMSec '21), June 22-25, 2021, Virtual Event, Belgium. ACM, New York, NY, USA, 11 pages. https://doi.org/10.1145/3437880. 3460412

\section{Introduction}

Video conferencing (VC) systems utilize text, images, audio and video and other media to enable communication. They are used to an increasing degree by organisations and private persons, especially considering the trend of social distancing during the COVID19 pandemic ${ }^{1}$. The increased usage is followed by an rising need to evaluate and understand security and privacy in these video conferencing system.

The meta and media data send during video conferencing session contains various events that can be used to identify or differentiate users and their behavior. Such information can be highly sensitive and needs proper protection. To evaluate whether and how this sensitive data can be identified within the network data streams observable during VC sessions, our paper proposes a systematic approach. As an example, the video conferencing service Zoom $\left(V C S_{A}\right)$ with Client Version 5.4.57862.0110 [4], the mobile Android App Client Version 5.5.2 (1328) [4], the webbased client and the Zoom Conferencing servers (accessed between Jan 21st and Feb 4th) is selected for investigations into media and network data streams. However, our approach can be applied to other video conferencing solutions $\left(V C S_{*}\right)$. Our approach aims at identifying and gathering the contents of (potentially) encrypted network data streams containing multimedia and VC-related meta data. This data is investigated in order to identify discernable information about the participants of the conference using various methods based on the type of data contained within the respective network data streams. Information

\footnotetext{
${ }^{1}$ https://www.uctoday.com/collaboration/video-conferencing/video-conferencingstatistics/, accessed 2021/02/25
} 
extracted without decryption of the respective data (plain data) and those obtained by decrypting the encrypted data (decrypted content) is aggregated to derive data with a potential harm to the users privacy.

The main contribution is the systematic approach to perform forensic analysis on the mmultimedia and VC-related meta data within the network data stream by combining the results from the investigation of plain data and decrypted content. Our approach is based on a forensic process model and encompasses techniques from computer forensics and media forensics in particular, including pattern recognition for speaker identification. Motivated by [3], our paper introduces a generic network setup to enable systematic forensic investigations including pattern recognition to identify distinct users.

Previous work conducted hard drive forensics in $V C S_{A}$ [9], or investigated security issues $[8,13]$. The main contributions of our paper include:

- Introducing an approach for a forensic framework to investigate plain data and encrypted content representing multimedia and VC-related meta data within the network data streams in video conferencing.

- Developing a generic investigative setup with different networks, clients and proxies for the investigation of video conferencing sessions.

- Applying the framework to $V C S_{A}$. The investigation leads to the discovery of 20 possible user identification and differentiation features, with 17 applicable to the VC-related meta data of encrypted traffic in $V C S_{A}$.

- Showing that $V C S_{A}$ End-to-End-Encryption (E2E) does not provide additional protection against identification and differentiation of users and their behavior in the sessions.

- Training a pattern recognition classifier that is able to identify users based on features extracted from encrypted audio stream.

In the following, section 2 provides some selected background. Section 3 presents a forensic approach to examine network communications within video conferencing network traffic. Section 4 applies this approach to $V C S_{A}$ video conferencing. Section 5 discusses the findings and compares the results with existing work [6]. In section 6, the results are summarized and further work is motivated.

\section{Background}

This section provides a short introduction into the forensic process model which forms the foundation for the systematic approach presented within in this work. In addition, an overview on the topic of speaker identification in the encrypted domain is provided. Furthermore, an overview on the preceding research into the used example $\left(V C S_{A}\right)$ and definitions are provided.

\subsection{Computer Forensic Approach}

Computer forensics relies on scientifically derived and proven methods for the purpose of reconstructing events (see [10]). Such an approach implies the use of proven methods in a structured process in order to document various pieces of information with the overall aim to present them in a manner which enables a person reviewing the result to form a well-funded conclusion based on this information. The approach presented within our paper has the overall aim to enable a person to achieve a well-funded conclusion based on the findings. Hence, a scientific and well-structured approach from the fields of computer forensics is applied.

Our approach follows a structured process as often described in various models of the forensic process. In our paper, we use the forensic process as described in [1] based on [7].

This forensic process model structures the forensic process along the lines of six Investigation Steps:

Strategic Preparation (SP): Strategic measures taken in an IT-system in order to support a forensic investigation prior to an incident.

Operational Preparation (OP): Operational measures of preparing a forensic investigation after a suspected incident.

Data Gathering (DG): Measures to acquire and secure digital evidence.

Data Investigation (DI): Measures to evaluate and extract data for further investigation.

Data Analysis (DA): Measures for detailed analysis and correlation of digital evidence from various sources.

Documentation (DO): Measures for the detailed documentation of the proceedings (Process Accompanying Documentation) and for the compilation of a report on the incident (Final Documentation).

This forensic process model also differentiates between nine Data Types based on how the respective data is handled during the forensic process (see 1):

Raw data $\left(D T_{1}\right)$ : A sequence of bits within the Data Streams of a computing systems not (yet) interpreted.

Hardware data $\left(D T_{2}\right)$ : Data in a computing unit not, or in a limited way, influenced by software.

Details about data $\left(D T_{3}\right)$ : Data added to other data, stored in annotated chunks of data or externally.

Configuration data $\left(D T_{4}\right)$ : Data changed by software and which modifies the behavior of software and hardware, excluding the communication behavior.

Network configuration data $\left(D T_{5}\right)$ : Data that modifies system behavior with regards to communication.

Process data $\left(D T_{6}\right)$ : data about a running software process within a computing unit.

Session data $\left(D T_{7}\right)$ : data collected by a system during a session, which consist of a number of processes with the same scope and time frame.

Application data $\left(D T_{8}\right)$ Data representing functions needed to create, edit, consume or process content relied to the key functionality of the system.

Functional data $\left(D T_{9}\right)$ : data content created, edited, consumed or processed as the systems key functionality.

\subsection{Activity and User Identification}

Identification of activity or even distinct users based on (encrypted) multimedia data has been explored in multiple publications and is a known privacy threat: An example for activity identification 


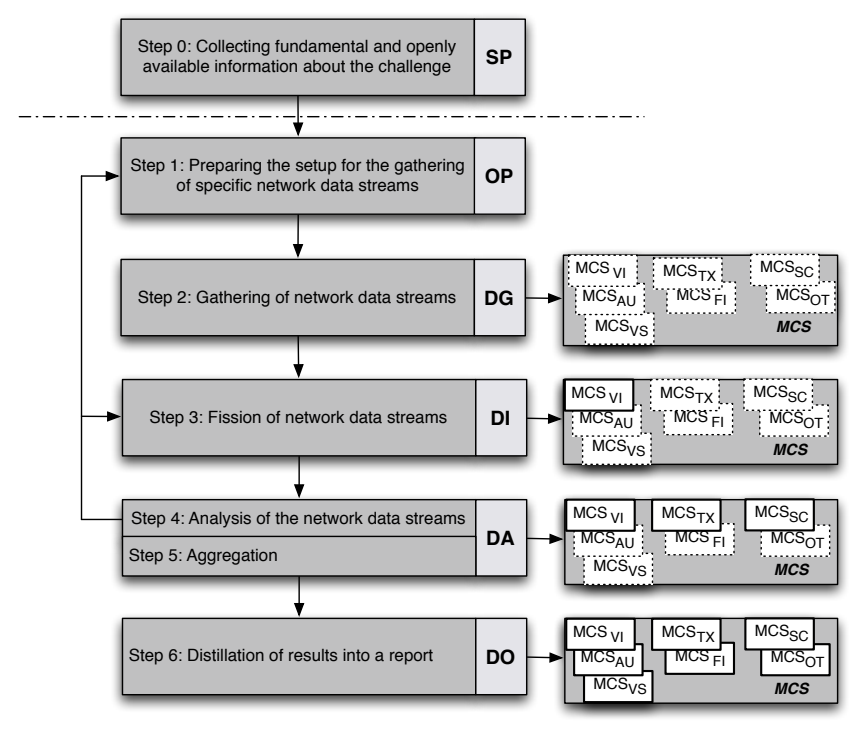

Figure 1: The forensic approach to investigate video conferencing solutions consists of $1+5$ steps. In DG, DI, DA and DO different media streams $(M C S)$ are gathered, fissioned and information extracted from them and then analysed.

can be found in [12] where bits/second (bitrate) is used as a feature to identify events of change during a video media stream. Neural networks have been used in [15] to explore the possibility to distinguish between different voice commands in the encrypted audio stream originating from smart speakers. A similar approach has been presented in [17], where hidden Markov Models were used to identify specific phrases within encrypted audio streams. A more recent approach presented in [3] uses bits/second as a feature to distinguish between different user activities (usage of webcam or microphone) within the encrypted multimedia streams. User identification based on encrypted multimedia streams has been explored e.g. in [16] which uses the timing between various packets in an encrypted (TLS) text stream as a feature to extract a keystroke behavior in order to identify distinct users.

\subsection{Example for a Video Conferencing Software: $V C S_{A}$}

To apply our approach to $V C S_{A}$, a short overview on into this video conferencing solution is provided and the multimedia streams are defined.

$V C S_{A}$ is a video conferencing solution that is accessed through the use of a client application installed on a machine or running in a browser. The backend application is typically executed as a cloud service. However, an on-premise solution is also available ${ }^{2}$ keeping the video and audio content on the on-premise servers (hosted by the customers) while contact and meta data is still send to and through the providers cloud. Multiple security issues where reported (see e.g. [5] and [14]).

\footnotetext{
${ }^{2}$ https://support.zoom.us/hc/en-us/articles/360034064852-Zoom-On-PremiseDeployment, accessed 2021/01/21
}

Beside the primary VC functionality of $V C S_{A}$ other functionality outside of those sessions are available (like text chats and scheduling, which both were already analyzed in [9]). Our paper focuses on the video conferencing sessions themselves. The following multimedia streams $(M C S)$ are defined:

$M C S_{A U}:$ Audio (continuous)

$M C S_{V I}$ : Video (continuous)

$M C S_{S C}:$ Screensharing (continuous)

$M C S_{V S}$ : Sharing of Video (continuous)

$M C S_{T X}:$ Text (chat) (spatial)

$M C S_{F I}:$ File transfer (spatial)

$M C S_{O T}$ : Other spatial streams

$V C S_{A}$ clients are available for various platforms, including desktop applications (Windows, MacOS, Linux), as mobile applications (Android, iOS) and a browser-based application. The used browserbased application does not support some features including $\mathrm{E}_{2} \mathrm{E}^{3}$. The exact codecs used for the transmission of the audio and video stream are unknown but based for instance on H.264. ${ }^{4}$ the provider encrypts all network traffic (see [9]). Whenever the term encrypted is used in our paper, it refers to this transport encryption (TLS). In the case the E2E feature is used this is explicitly stated.

\section{Forensic Approach to Examine Network Data Streams In Video Conferencing}

Video conferencing in general follows the structure as presented in figure 2. Various Clients $\left(\mathrm{CL}_{1}\right.$ to $\left.\mathrm{CL}_{n}\right)$ exchange multimedia data (in the forensic process model described as $D T_{9}$ ), utilizing a dedicated server (referred to as multimedia server - MMS). Since it might be possible that the different multimedia streams are handled by different network data streams, the term multimedia services is used in this case. Additional VC-related meta data is exchanged with the infrastructure. This meta data is utilized for user or session management, scheduling, activity logging or other means $\left(D T_{4}\right.$, $\left.D T_{5}, D T_{7}\right)$. This infrastructure is referred to as infrastructure services. These services might be distributed among different servers $\left(\mathrm{IS}_{1}\right.$ to $I_{N}$ ). Each of the connections to this services is a specific network data stream although the distinction between the respective streams might be difficult. All these instances of communication are usually encrypted.

This leads to an approach for a systematic investigation based on the forensic principles (see section 2.1):

(SP) Step 0: OSINT information collection about the challenge

In this first step, fundamental information about the investigation target is gathered. This includes fundamental information about the setup, the provided functionality in terms of VC-related meta data and multimedia $(M C S)$ streams and an overview on the research already conducted into the respective platform.

(OP) Step 1: Setting up the collection of specific network data streams

An investigative setup to gather the network data streams to all infrastructure servers $\left(I S_{1}\right.$ to $I S_{n}$, for VC-related meta data) and

\footnotetext{
${ }^{3}$ https://www.wired.com/story/how-to-enable-zoom-encryption/, accessed 2021/02/18

${ }^{4}$ https://support.zoom.us/hc/en-us/articles/202445433-Conference-Room-

Connector-Supported-Devices, accessed 2021/02/26
} 

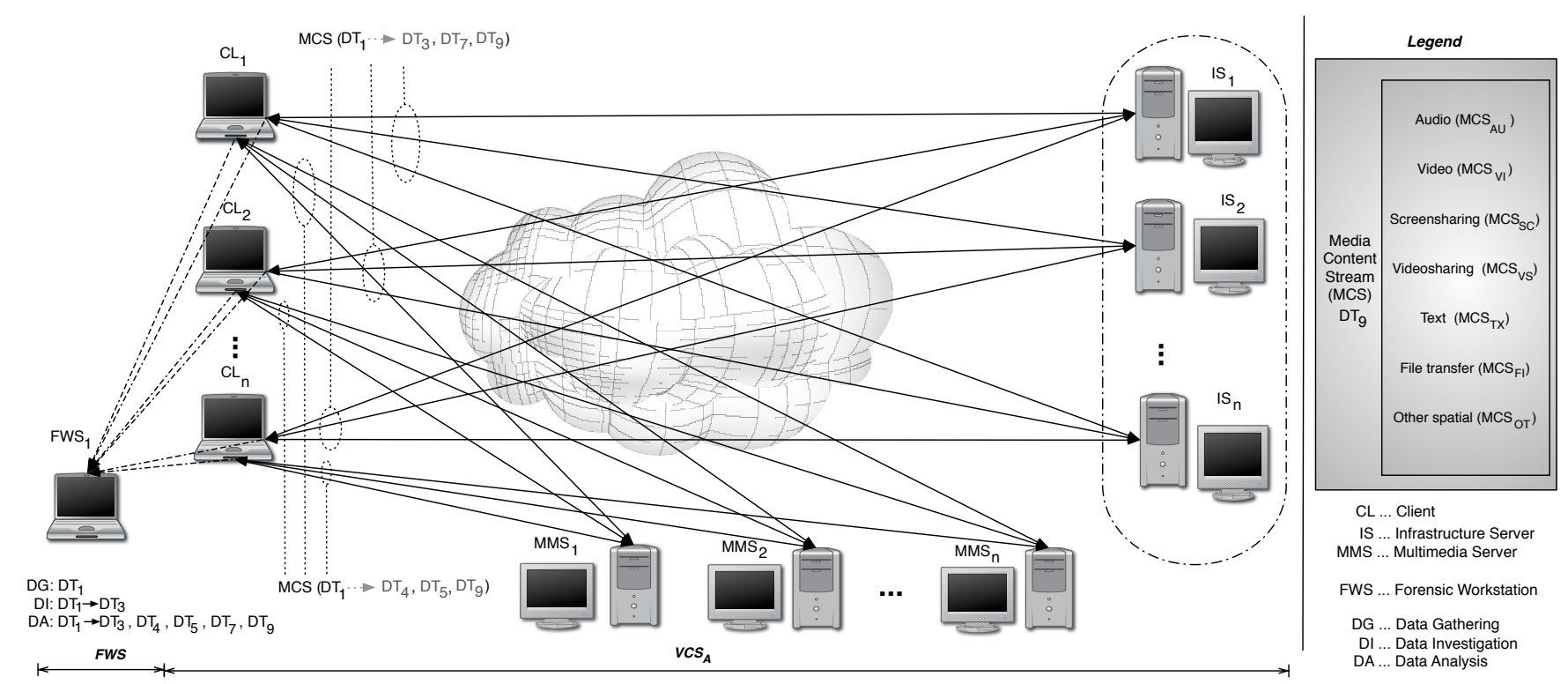

Figure 2: Generalized landscape of communication in VC sessions. Different clients $\left(C L_{1}\right.$ to $\left.C L_{n}\right)$ exchange VC-related meta and multimedia data with infrastructure servers $\left(I S_{1}\right.$ to $\left.I S_{n}\right)$ and multimedia servers $\left(M M S_{1}\right.$ to $\left.M M S_{n}\right)$. The media streams of different types $(M C S)$ can be captured in transfer to and from the servers.

multimedia servers $\left(M M S_{1}\right.$ to $M M S_{n}$, mainly for multimedia data) is established. This relies on knowledge about the individual servers as shown in figure 2 .

(DG) Step 2: Acquisition of network data streams

The setup created during Step $\mathbf{1}$ is used to gather the specific network data streams.

(DI) Step 3: Fission of network data streams

This step applies the knowledge obtained during previous iterations of the process and covers the actions necessary to fissure the different network data streams gathered during Step 2.

(DA) Step 4: Analysis of network data streams

During this step, the respective network data streams are analysed using three different activities:

Step 4A: Discovery of newly identified network data streams This step aims at identifying encrypted and formerly not discovered network data streams. This identification can be based on different hostnames, IP addresses, port numbers, timing or other information. Depending on the situation, a fission of the collected network data into the newly identified network data streams is possible or need to be conducted during the next iteration of the process.

Step 4B: Identification of contents of the respective network data streams

The respective network data streams are investigated for contents which contain information about the observed client. The exact methods differ whether VC-related meta or multimedia data is investigated based on the data types of the forensic process model described in 2.1. This includes $D T_{3}, D T_{4}, D T_{5}, D T_{7}$ and $D T_{9} . D T_{3}$ and $D T_{7}$ are contained within the network data streams to the MMS $D T_{4}$ and $D T_{5}$ are also contained with the network data streams to the $M M S$ and control the client behavior with regard to functionality and communication. $D T_{9}$ is only available in the data streams to the the $M M S$ while all other $D T_{n}$ are also part of the network data streams to the IS.

Step 4C: Identification of contents which contain information about other Clients

Step 4B is repeated with a focus on contents which contain information about other clients partaking in the VC.

(DA) Step 5: Aggregation The results obtained during Step 4 are aggregated, creating a timeline of correlated events from the encrypted network data stream as well as plain text information, to provide an overview on the observations during the assessment. If the identification of additional network data streams during Step 3A a repetition of Step $\mathbf{1}(\mathbf{O P})$ is necessary. Hence the overall process is iterative.

(DO) Step 6: Documentation The various results are distilled into report as part of the DO during the forensic process. In the case of our investigation it is reported in the form of this scientific paper.

The process (figure 1) in itself is iterative and might require various passes as new respective data streams might be identified during Step 4A with the setup for the collection of these network data streams being insufficient to perform Step $4 B$ and Step $4 C$ in an exhaustive manner.

\section{Applying the Forensic Approach to $V C S_{A}$}

The forensic approach relies on general preparation (Step 0) and on multiple iterations of the succeeding steps in order to identify the greatest possible extent of data to analyze. Hence, Step $\mathbf{1}$ is refined multiple times and Step 2, Step 3 and Step 4 repeated multiple times to obtain the best investigative results. Whenever a new type of user identification, differentiation or event is identified, 


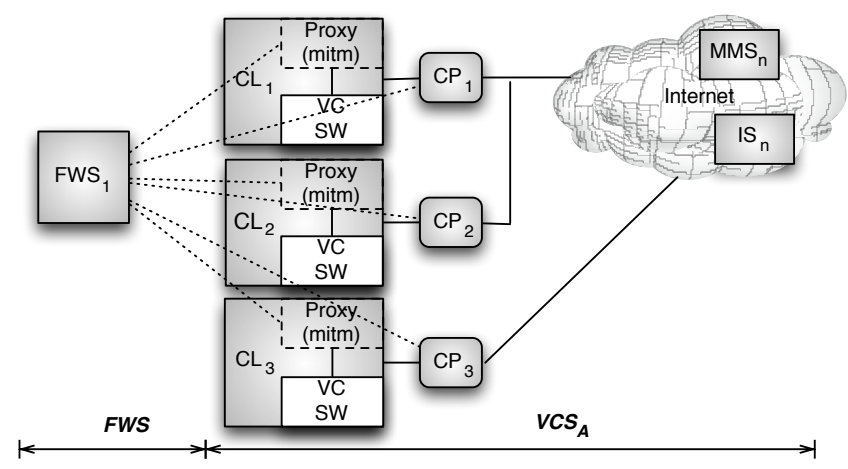

Figure 3: Investigative setup for the application of the forensic approach to video conferencing solution showing various Capturing Points $\left(C P_{n}\right)$

the finding is marked as an event $\left(E_{x}\right)$. Table 6 provides an overview on our 20 findings.

(SP) Step 0: Collecting fundamental information A preparatory step collects information about $V C S_{A}$ including general information, a review of the specific functionality (in terms of available multimedia streams and the specific events associated with these multimedia streams) and various research conducted into the respective solution. The collection and results of this step are presented in section 2.3.

\section{1 (OP) Step 1: Setting up the Collection of Specific Network Data Streams}

Initially a setup containing three distinct $V C S_{A}$ clients $\left(C L_{1}, C L_{2}\right.$ and $\left.C L_{3}\right)$ is established as shown in figure 3. Two of those clients $\left(C L_{1}\right.$ and $\left.C L_{2}\right)$ use the same internet connection while $C L_{3}$ uses a different one. This is due to the potential impact of the connection properties on features potentially useful for the application of biometric speaker recognition (see section 4.4.3). The different clients can be configured to use a specific proxy in order to gain insight into the encrypted content within the network data stream if necessary. Here, the OWASP $\mathrm{ZAP}^{5}$ or the mitmproxy ${ }^{6}$ can be employed. This setup allows for various points to capture network data streams (namely at $C P_{1}, C P_{2}$ and $C P_{3}$ ) and for flexibility with regards to various possible configurations. A forensic workstation $\left(F W_{1}\right)$ is employed to collect the network data streams (from $C P_{1}$ through $C P_{n}$ ) and the decrypted contents (from the Proxies in $C L_{1}$ through $C L_{n}$ ).

\section{2 (OP) Step 2: Gathering of Network Data Streams}

This setup is implemented by installing Manjaro Linux ${ }^{7}$ and $V C S_{A}$ Client Version 5.4.57862.0110 [4] on $C L_{1}$ and $C L_{2}$ and Windows 10 Pro with the same client on $C L_{3}$. On $C L_{3}$ the browser-based $V C S_{A}$ client and an Android client Android App Client Version 5.5.2 (1328) [4] is used in supporting investigations (aimed at confirming

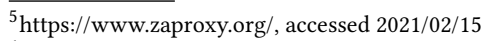

${ }^{6} \mathrm{https} / / /$ mitmproxy.org/, accessed 2021/02/15

${ }^{7}$ https://manjaro.org/, accessed 2021/02/15
}

specific investigation results). Wireshark Version $3.4 .3^{8}$ is used to capture the network data streams (at $C P_{1}, C P_{2}$ and $C P_{3}$ ). In addition, the OWASP ZAP is configured in order to investigate encrypted content. For this, a root CA is installed on the machines and the system wide use of the proxy server is configured ${ }^{9}$.

As expected, an influence of configuration parameters is detected during Step 4A leading to different investigative setups. These configuration parameters are:

AT: The account type of the host can be private (free, $A T_{P R}$ ), educational $\left(A T_{E D}\right)$ or educational on-premise $\left(A T_{O P}\right)$.

FW: A firewall blocking all non-DNS UPD traffic is enabled $\left(F W_{e n}\right)$ or disabled $\left(F W_{d i}\right)$.

PR: The traffic is routed $\left(P R_{e n}\right)$ or not routed $\left(P R_{d i}\right)$ through an HTTP proxy.

EE: The E2E functionality is enabled $\left(E E_{e n}\right)$ or disabled $\left(E E_{d i}\right)$. CL: The desktop client $\left(C L_{D E}\right)$, browser client $\left(C L_{B R}\right)$ or mobile client $\left(C L_{M B}\right)$ are used.

This step provides the network data streams $\left(D T_{1}\right)$ for the following analysis.

\section{3 (DI) Step 3: Fission of Network Data Streams}

This step encompasses the extraction of the VC-related meta data and the multimedia data from the overall network capture. Practically, various prepared filters are applied to extract the respective data in an automated manner using Wireshark. In the terms of the forensic process model, DI is conducted by extracting $D T_{3}$ (details about data) and $D T_{9}$ (functional data) from $D T_{1}$ (raw data). The identification and extraction of different kinds of data is restricted by the presence of encrypted content which can only partly be decrypted.

As shown in [3], video and audio streams can be extracted by filtering for the used UDP ports. The results of our paper confirm this observation with most configuration parameters:

$A T_{P R}$ or $A T_{E D}$ : An array of different $M M S$ to provide $M C S$ is used. During a session, one client uses the same $M M S$ for all MCS except $M C S_{F I}$. Different $C L_{D E}$ in the same session might use the same $M M S$ while and $C L_{D E}$ and $C L_{B R}$ in the same session use different $M M S$. This suggests an exchange of multimedia between the different $M M S$.

$F W_{d i}$ and $C L_{D E}$ : The multimedia streams are transmitted using UDP. The server-side UDP port is usually $8801^{10}$. The clientside UDP port is randomly assigned. Different MCS are usually assigned different UDP ports which allows for the distinction of media streams on the network level (see figure 4). In some cases, the clients changes the respective UDP ports during the sessions to consolidate all primary media streams to one UDP port. What triggers this behavior is unknown. $M C S_{S C}$ differs from $M C S_{A U}$ and $M C S_{V I}$ as the used UDP ports might change during one session. It is possible to use a video optimization functionality to use share videos $\left(M C S_{V S}\right)$ running on the clients machine. In case this functionality is used while no other $M C S_{V I}$ is active, the video data will be send over a different port. In case at least one other $M C S_{V I}$ is activated, it will be send together with $M C S_{V S}$ over the same port. The audio

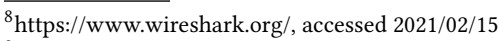

${ }^{9}$ Zoom only accepts proxies on port 443 .

${ }^{10} \mathrm{With}$ rare exceptions triggered by unkown reasons
} 
stream of a shared video will always be send together with $M C S_{A U}$ and cannot be separated. $M C S_{T X}$ and $M C S_{O T}$ are transferred via non-HTTP TLS traffic preventing a distinction based on port numbers due to the use of the same TCP port. However, an extraction of information based on the plain data is possible. The transmission of $M C S_{T X}$ causes a short spike in the transferred bytes as an example. The size of the spike grows with the amount of $M C S_{T X}$ transmitted.

$F W_{e n}$ : All MCS are routed through an HTTP Tunnel. A separation remains possible since the different $M C S$ are using different TCP ports.

$E E_{e n}$ : A separation of $M C S$ based on UDP ports is possible. However, in some cases $M C S_{A U}$ and $M C S_{V I}$ are transmitted using the same stream ${ }^{11}$. This behavior points towards the presence of $D T_{5}$ within the network communication. This includes signals which control the flow of network data, e.g. the changing of network ports during a session.

$C L_{B R}$ : Every $M C S$ uses a different websocket with an unique client port. Furthermore, the extraction of decrypted contents from all MCS is possible ${ }^{12}$.

\section{4 (DA) Step 4: Investigation of the Respective Network Data Streams}

During this step, the respective network data streams are identified and analysed (DA within the forensic process model) in three different substeps:

4.4.1 Step 4A: Discovery of newly identified network data streams The identification of the network data streams relies on a review of the respective network connections in the captured network traffic. These connections contain combinations of source and destination addresses as well as different source and destination ports point towards a range of different services, including infrastructure services $\left(I S_{1}\right.$ to $\left.I S_{N}\right)$ as well as multimedia services $\left(M M S_{1}\right.$ to $\left.M M S_{N}\right)$.

This is complicated in the case of $V C S_{A}$ due to the fact that the network data streams differ wildly depending on the configuration parameters chosen (see section 4.2): $V C S_{A}$ has the ability to use fallbacks to enable connectivity. These techniques include measures like HTTP-Tunneling, protocol switching from UDP to TCP, usage of WebRTC and/or Websockets ${ }^{13}$. This increases the analysis complexity but can be overcome by the application of our systematic approach.

The influence of the configuration parameters requires a series of different investigative setups with varying parameters in order to identify all separable $M C S$. The characteristics used to separate the various $M C S$ are summarized here:

$M C S_{A U}:$ Distinct port (UDP or TCP)

$M C S_{V I}:$ Distinct port (UDP or TCP)

$M C S_{S C}:$ Distinct port (UDP or TCP)

$M C S_{V S}:$ Distinct port (UDP or TCP)

$M C S_{T X}:$ TCP peak

\footnotetext{
${ }^{11}$ For example: the audio stream is activated and uses a distinct port. As soon as the video is activated in addition, the audio stream switches to the same port as the video stream. When the video stream stops, the audio stream switches back to the original port.

${ }^{12}$ by using the Browser Developer Tools

${ }^{13}$ https://zoom.us/docs/doc/Zoom\%20Connection\%20Process\%20Whitepaper.pdf, accessed 2021/02/15
}

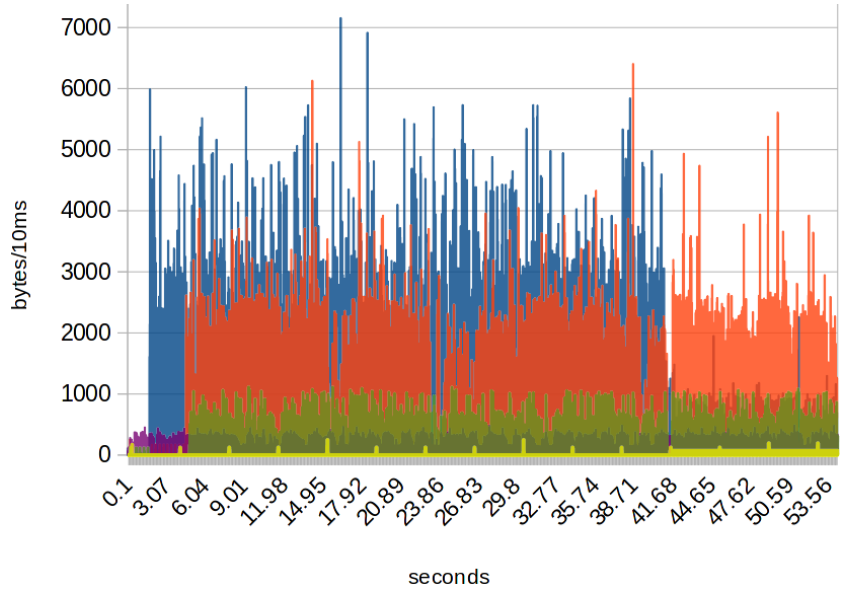

Figure 4: Plotted throughput from Wireshark capture. Most $M C S$ identified in Step 3 and Step 4 a can be clearly distinguished by the used UDP ports. Blue: Video stream $\left(M C S_{V I}\right)$ out. Orange: Video stream $\left(M C S_{V I}\right)$ in. Purple: Audio stream $\left(M C S_{A U}\right)$ out. Green: Audio stream $\left(M C S_{A U}\right)$ in. Yellow: Screensharing stream $\left(M C S_{S C}\right)$ out.

$M C S_{F I}:$ Different $M M S$ than the other $M C S$ $M C S_{O T}:$ (Partly) distinct TCP peaks

4.4.2 Step 4B: Identification of contents of the respective network data streams After the various streams are identified and seperated they are analyzed. At first, the findings in multimedia data are discussed before those in VC-related meta data are discussed.

Multimedia Content Streams The MCS offer the means to identify a series of events during the use of $V C S_{A}$ (as shown in figure 6). This is already presented in [3] and is enriched by additional observations as follows:

$V C S_{A}$ adapts the $M C S$ to the current user behavior. For example, $C L_{D E}$ stops receiving $M C S_{V I}$ when the user minimizes the clients window $\left(E_{9}\right)$. In the case of $C L_{B R}$ only one users video stream can be shown at any given time - and only the video stream from this user is received. Based on this behavior, the presence of $D T_{4}$ representing signals to stop the transmission in $M C S_{V I}$ seems reasonable. Currently this $D T_{4}$ is not accessible due to encryption (encrypted content).

The throughput of the respective $M C S$ can be used to identify user behavior $\left(E_{4}, E_{5}\right)$. In the case of $M C S_{S C}$, the sharing of a static screen results in significantly less network traffic than an interactive screen $\left(E_{20}\right)$. This is in line with the results of [3] where the impact of certain user interactions on throughput was shown. However, the findings point towards an increase in throughput due to an increased amount of activated webcams from [3] could not be performed, albeit short peaks in throughput are identifiable whenever a new webcam is activated.

Whenever a user joins or leaves a session a peak in all streams is observable $\left(E_{2}, E_{3}\right)$.

This extraction of user behavior is also possible for the secondary media streams: Every send text messages in $M C S_{T X}$ leads to a short peak with regards to the transferred TLS packets and bytes $\left(E_{13}\right)$. 
The longer the text, the larger the peak. $V C S_{A}$ features so called reactions. These indicate the current participant status with an emoji. Setting a reaction is contained within $M C S_{T X}$ and can be distinguished from text messages by the small size of the transferred packets and bytes $\left(E_{18}\right)$.

$M C S_{F I}$ is used to transfer files from the local file system. The files are send and received with an HTTP call. The transfer can be separated from other MCS by using a specific $M M S$ : files.zoom.us $\left(E_{14} \cdot E_{15}\right)$. During one session, different $M M S$ for the file transfer might be used (same domain, different IPs). The content of the files is obfuscated (or encrypted) before it is send in any case $\left(E E_{d i}\right.$ and $\left.E E_{\text {en }}\right)$. The exact functionality of this obfuscation is unknown but seems do be dynamic, since the content differs in every transfer, even if the same file is transferred multiple times. The meta data of the file (name and type) is send non-obfuscated to the $M M S$ $\left(E_{17}\right)$. The size of the file can be calculated exactly $\left(E_{16}\right)$, since every obfuscated byte represents one byte of the file.

$V C$-related Meta data With $A T_{P R}$, extensive log files are send to $I S$ servers after every session ends. These logs contain user behavior information during the session, including every activation/deactivation of microphone and webcam.

Connections In the case of $C L_{B R}$, all MCS are exchanged with $M M S_{1}$ through $M M S_{n}$ in Amazons AWS cloud (e.g. with hostname ec2-18-159-166-167.eu-central-1.compute.amazonaws.com. In the case of $A T_{E D}$ or $A T_{O P}$ and $P R_{e n}$, twelve connections to eleven different $I S$ can be found during the startup and the ongoing session (see table 1). Every connection to a IS controls a certain aspect of the session. In case of $A T_{O P}$, the $M M S$ server have custom IP addresses. In case of $A T_{E D}$ or $A T_{P R}$, the $M M S$ reside in *.cloud.zoom.us in both cases. $V C S_{A}$ published a list of all the IP addresses a client might connect to ${ }^{14}$. However, the list seems to be incomplete, since the investigated clients connected to servers not listed (e.g. 130.224.220. 191, 130.61.165.176).

Summary The network streams allow for the identification of 20 distinct events ( $E_{1}$ through $E_{2} 0$, see table 6 ) within VC sessions utilizing $V C S_{A}$ based on $D T_{9}$ within $M C S$. The findings points towards the conclusion of $V C S_{A}$ optimizing the amount of transferred network data in order to reduce the load on its servers and for the clients. This leads to a potential leakage of information of user behavior or about the session itself: (1) $V C S_{A}$ does recognize whether a user has the client/ browser window in the foreground or not. In case it is in the background (not visible), the client will stop to receive $M C S_{V I}, M C S_{S C}$ and $M C S_{V S}$. The same is true in case the video is not visible inside the client $\left(E_{9}\right)$. (2) Additionally, the client stops sending the $M C S_{V I}$ in case no other participant can see the video (either because nobody has the application in the foreground, or the speaker is not visible inside the app). Therefore, it is possible for every participant to derive whether at least one other participant is watching the $M C S_{V I}$ or not (paying attention, or not, E19). From this observations it can be concluded that the client is sending some kind of event to the server in order for the server to regulate in- and outgoing $M C S_{V I}$. Therefore, it is possible

\footnotetext{
${ }^{14}$ https://support.zoom.us/hc/en-us/articles/201362683-Network-Firewall-or-ProxyServer-Settings-for-Zoom, accessed 2021/02/22
}

\begin{tabular}{|c|c|c|c|c|}
\hline Connection & Account & Client & Proxy & Purpose \\
\hline$\overline{130.61 . .^{* *}}$ & $A T_{E D}$ & $\begin{array}{l}C L_{D E} \\
C L_{M O}\end{array}$ & $x$ & $\begin{array}{l}M M S \text { for transfer of } \\
M C S\end{array}$ \\
\hline $130.224 .^{* *}$ & $A T_{E D}$ & $\begin{array}{l}C L_{D E} \\
C L_{M O}\end{array}$ & $x$ & $\begin{array}{l}M M S \text { for transfer of } \\
M C S\end{array}$ \\
\hline *.cloud.zoom.us & $\begin{array}{l}A T_{E D} \\
A T_{P R}\end{array}$ & $\begin{array}{l}C L_{D E} \\
C L_{M O}\end{array}$ & $x$ & $\begin{array}{l}M M S \text { for transfer of } \\
M C S\end{array}$ \\
\hline $\begin{array}{l}\text { *.amazonaws. } \\
\text { com }\end{array}$ & $\begin{array}{l}A T_{E D} \\
A T_{P R}\end{array}$ & $C L_{B R}$ & $\checkmark$ & $\begin{array}{l}M M S \text { for transfer of } \\
M C S, I S\end{array}$ \\
\hline $\begin{array}{l}\text { ([x])logfiles. } \\
\text { zoom.us }\end{array}$ & any & $\begin{array}{l}C L_{D E} \\
C L_{M O}\end{array}$ & $\checkmark$ & $I S$, Session Events \\
\hline async.zoom.us & $A T_{E D}$ & $\begin{array}{l}C L_{D E} \\
C L_{M O}\end{array}$ & $\checkmark$ & $I S$, Obfuscated \\
\hline $\begin{array}{l}\text { contac } \\
\text { zoom.t }\end{array}$ & $\begin{array}{l}A T_{E D} \\
A T_{P R}\end{array}$ & $\begin{array}{l}C L_{D E} \\
C L_{M O}\end{array}$ & $\checkmark$ & $\begin{array}{l}\text { IS, Other Users Contact } \\
\text { Data }\end{array}$ \\
\hline $\begin{array}{l}([\mathrm{x}]) \mathrm{zwww} 3 . \\
\text { zoom.us }\end{array}$ & $A T_{E D}$ & & $\checkmark$ & $I S$, Settings of Session \\
\hline $\begin{array}{l}\text { ([x])zpns.zoom. } \\
\text { us }\end{array}$ & Any & $\begin{array}{l}C L_{D E} \\
C L_{M O}\end{array}$ & $\checkmark$ & $\begin{array}{l}\text { IS, Token exchange via } \\
\text { Websocket }\end{array}$ \\
\hline $\begin{array}{l}\text { ([x])xmpapi. } \\
\text { zoom.us }\end{array}$ & Any & $\begin{array}{l}C L_{D E} \\
C L_{M O}\end{array}$ & $\checkmark$ & $\begin{array}{l}I S \text {, XMPP information, } \\
\text { Cert exchange }\end{array}$ \\
\hline zoom.us & Any & $\begin{array}{l}C L_{D E} \\
C L_{M O}\end{array}$ & $\checkmark$ & $I S$, UI information \\
\hline zoom. & & & $\checkmark$ & JS libs and UI information \\
\hline $\begin{array}{l}\text { keyserver- } \\
\text { us.zoom.us }\end{array}$ & $\begin{array}{l}A T_{E D} \\
A T_{O P}\end{array}$ & $\begin{array}{l}C L_{D E} \\
C L_{M O}\end{array}$ & $\checkmark$ & $I S$, E2E Keys \\
\hline $\begin{array}{l}\text { tunnel[x].zoom. } \\
\text { us }\end{array}$ & $A T_{E D}$ & $\begin{array}{l}C L_{D E} \\
C L_{M O}\end{array}$ & $x$ & IS, HTTP Tunnel \\
\hline $\begin{array}{l}\text { zoomny }[\mathrm{x}] \mathrm{ht} . \\
\text { zoom.us }\end{array}$ & $A T_{E D}$ & $\begin{array}{l}C L_{D E} \\
C L_{M O}\end{array}$ & $x$ & IS, HTTP Tunnel \\
\hline $\begin{array}{l}\text { us[x]as.zoom. } \\
\text { us }\end{array}$ & $A T_{P R}$ & $\begin{array}{l}C L_{D E} \\
C L_{M O}\end{array}$ & $\checkmark$ & $I S$, Obfuscated user data \\
\hline $\begin{array}{l}\text { us[x]file.zoom. } \\
\text { us }\end{array}$ & $\begin{array}{l}A T_{P R} \\
A T_{E D}\end{array}$ & $\begin{array}{l}C L_{D E} \\
C L_{M O}\end{array}$ & $\checkmark$ & $\begin{array}{l}M M S \text {, Sending files } \\
\left(M C S_{F I}\right)\end{array}$ \\
\hline
\end{tabular}

Table 1: Overview on the connections to $M M S$ and $I S$ in $V C S_{A}$ during a session. $A T$ : Observable with a specific account type. $C L$ : Observable with a specific client. Proxy: Observable in HTTP Proxy. Purpose: Type of network traffic and server type (MMS: Multimedia server, $I S$ : Infrastructure server) [x]: Any number. $([\mathbf{x}])$ : Any number or nothing. $M M S$ : Multimedia server. Fewer connections for $C L_{B R}$ result from (1) no E2E exists and (2) session, configuration and user data is fully transmitted to the 1 st party server.

to deduct at which time which participant is paying attention based solely on plain data.

Our investigation discovered no major differences between $A T_{E D}$ and $A T_{O P}$. All VC-related meta data (e.g. user data, log files, configuration data) is transmitted to $I S$ belonging to $V C S_{A}$ US servers. The on-premise servers are only used as $M M S^{15}$.

Although minor differences in the media streams exist between $E E_{e n}$ and $E E_{d i}$, the means of separation and the revealed information are available in both scenarios. In regards to this information, E2E does not offer additional protection.

$C L_{M B}$ establishes the same connections and shows the same behavior as $C L_{D E}$. The only difference is that the mobile client

\footnotetext{
${ }^{15}$ This is exactly how $V C S_{A}$ itself describes the on-premise solutions.
} 


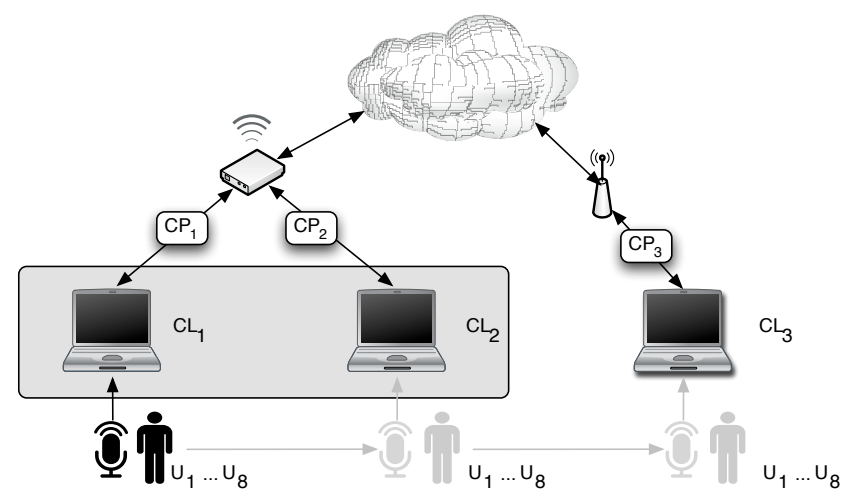

Figure 5: The investigation includes three $C L_{n}$ in two different networks and three corresponding capturing points $\left(C P_{n}\right)$. All clients are connected to a microphone activated at only one $C L_{n}$ per time (per test). All eight users $(U)$ are using every microphone, one after another.

can only display one concurrent video stream. The clients are not further analyzed specifically.

4.4.3 Step 4C: Identification of contents which contain information about other Clients The network data streams also allow the observer to obtain information about other clients participating in a conference. This section discusses the observable events relevant for remote clients.

Multimedia Content Streams The events described in section 4.4.2 can be used to obtain information about other clients during a $V C S_{A}$ session depending on the exact circumstance. E.g., if it is known that only one remote client is connected to a conference, the throughput of the received $M C S_{A I}$ indicates whether the microphone is muted, the speaker inactive or active. For $M C S_{V I}$, an increase in throughput indicates activity.

Further, the possibility to identify specific users within the $M C S_{A U}$ is explored. For this exploration, eight speakers are recruited ${ }^{16}$. The setup (figure 5) is a refinement of the setup from section 4.1. $C L_{1}$ and $C L_{2}$ use the same internet connection, while $C L_{3}$ is connected using a different connection. The network traffic is captured at every client (extraction points $A P_{1}, A P_{2}$ and $A P_{3}$ ). The configuration parameters $A T_{E D}, F W_{d i}, P R_{d i}, E E_{d i}$ and $C L_{D E}$ are used for all clients.

The speakers (users, $U$ ) read a set of random texts for 30 to 60 seconds at a given $C L_{n}$ and then move unto the next. During these recordings, all other $C L_{n}$ are muted and no actions are performed within the conference session. After these two texts are read, the speaker moves to the next client and the process starts over. Hence, any test is captured at one extraction point as outgoing traffic towards the $M M S$ used for $M C S_{A U}$ and at the other two extraction points as incoming traffic from the $M M S$ used for their $M C S_{A U}$. This setup is used to collect a total of 144 captures (8 Users $* 2$ Times repeated $* 3 C L(\mathbf{N}) * 3$ Extraction points) with some additional recordings for additional investigations to confirm the findings.

\footnotetext{
${ }^{16}$ Three female, five male, age range $20-40$, native German speakers.
}

\begin{tabular}{l|l|l|l|l|l|l|l|l} 
& $\mathrm{U} 1$ & $\mathrm{U} 2$ & $\mathrm{U} 3$ & $\mathrm{U} 4$ & $\mathrm{U} 5$ & $\mathrm{U} 6$ & $\mathrm{U} 7$ & $\mathrm{U} 8$ \\
$\mathrm{U} 1$ & 5632 & 0 & 297 & 0 & 0 & 1622 & 0 & 0 \\
$\mathrm{U} 2$ & 0 & 2788 & 0 & 0 & 5135 & 7 & 45 & 4 \\
$\mathrm{U} 3$ & 2549 & 0 & 7109 & 3 & 2581 & 145 & 0 & 0 \\
$\mathrm{U} 4$ & 0 & 961 & 0 & 1722 & 3992 & 998 & 8 & 0 \\
$\mathrm{U} 5$ & 0 & 0 & 4 & 1770 & 15 & 5624 & 987 & 2 \\
$\mathrm{U} 6$ & 0 & 17 & 0 & 4 & 2751 & 2621 & 3 & 2770 \\
U7 & 0 & 7 & 0 & 0 & 17 & 19 & 4996 & 2648 \\
U8 & 0 & 65 & 0 & 7 & 0 & 12 & 2654 & 5169
\end{tabular}

Table 2: Confusion Matrix for eight users. The Kappa statistic is 0.364 in this case.

\begin{tabular}{l|l|l|l|l|l} 
& $\mathrm{U} 1$ & $\mathrm{U} 3$ & $\mathrm{U} 5$ & $\mathrm{U} 6$ & $\mathrm{U} 7$ \\
$\mathrm{U} 1$ & 22255 & 302 & 0 & 0 & 0 \\
$\mathrm{U} 3$ & 394 & 36129 & 0 & 0 & 0 \\
$\mathrm{U} 5$ & 4 & 0 & 16043 & 6115 & 2915 \\
U6 & 0 & 8 & 8212 & 13115 & 2947 \\
U7 & 0 & 0 & 4977 & 4907 & 5592
\end{tabular}

Table 3: Confusion Matrix for five selected users. The Kappa statistic was $\underline{0.6826}$ in this case.

The main objective is to explore the possibility of identify specific users within the setup. For this, pattern recognition is used. Following the pattern recognition pipeline, the preprocessing is performed using network filters in order to separate the $M C S_{A U}$. The feature extraction is conducted with a purpose-built extractor based on the approach presented in [2]. The extractor uses a range of heuristic features which are calculated for windows of packets (either 1 second or 500 packets). These features include:

- Throughput in each direction (and ratio between both directions) in windows of packets,

- Packet size in each direction (min, max, mean, standard deviation in windows of packets),

- Entropy of the packets in each direction (min, max, mean, standard deviation in windows of packets),

- Ratio between bidirectional packets in packet windows.

The sets of extracted features (instances) are labelled in accordance to the specific questions. The goal is to analyze the extracted features in respect to speaker identification. The instances from the first repeat (T1) are used for training to build a model using J48 which is then applied to the instances from the second repeat (T2). The results are shown in table 2 .

Some distinction is possible within this setup while there are notable misclassifications. $U 1, U 3, U 7$ and $U 8$ could be clearly identified. $U 5$ and $U 6$ are difficult to distinguish from each other with the same behavior for $U 7$ and $U 8$.

Multiple recordings are conducted with a reduced amount of speakers. When the number of speakers are reduced to five or six, the achieved results are far better. The results obtained after removing $U 2, U 4$ and $U 8$ from the training and test sets and can be found in table 3 .

In this case only $U 7$ is difficult to identify. For various iterations with six distinct users, the Kappa statistic floats between 0.5 and 0.6 . 


\begin{tabular}{l|l|l|l} 
& Network $C L_{1}$ & Network $C L_{2}$ & Network $C L_{3}$ \\
Network $C L_{1}$ & 2935 & 5999 & 8 \\
Network $C L_{2}$ & 2582 & 9670 & 228 \\
Network $C L_{3}$ & 43 & 2401 & 9223
\end{tabular}

Table 4: Confusion Matrix for thee three client networks. The Kappa statistic was $\underline{0.4747}$ in this case.

In addition, the influence of the network connection on the classification results is investigated.

The results in table 4 show a great amount of misclassifications between $\mathrm{N} 1$ and N2. N3 can be clearly distinguished from both of them. This is plausible, since N1 and N2 share the same internet connection.

$V C$-related meta data With $C L_{B R}$, information about other users can easily be extracted from the encrypted content. The TLS-encrypted Websocket streams can be transformed to decrypted content by the use of the proxy.

Whenever a user joins a session its e-mail address ${ }^{17}$, username and ID $\left(E_{11}\right)$, as well as client type, operating system and other information $\left(E_{12}\right)$ are broadcast to all other clients. Every activation/deactivation of a webcam or microphone is also send as an event over Websockets. With $C L_{D E}$, no such observations were possible (since the decryption was not possible). However, since $C L_{D E}$ supports all user functionality of $C L_{B R}$, the corresponding information is most likely also broadcast.

4.4.4 (DA) Step 5: Aggregation The analysis of the VC-related meta and multimedia data leads to the identification of various events. Some events can be identified in both, VC-related meta and multimedia data and can be used to confirm the findings. Figure 6 shows the log entries (from VC-related meta data) and peaks in activity (from $M C S_{A U}$ ) both denote the unmuting of a participant. These events can be used to reconstruct a timeline of the overall session and the actions performed or to isolate sections where only a given speaker is active to apply speaker identification on this isolated section.

4.4.5 (DO) Step 6: Documentation The investigation is documented extensively, including the packet captures, test protocols and the resulting instances for machine learning resulting in our paper. Figure 6 summarizes all possibilities to identify or differentiate users and their behavior discovered in the previous steps.

\section{Discussion}

In this section the impact of the findings on the users privacy of videoconferencing solutions, validity threats and differences to a previous paper ([9]) are discussed.

\subsection{Impact on Users Privacy and Attack Scenarios}

Our forensic framework can be used to extract information which might harm users privacy. For an adversary it is possible to identify users by applying pattern recognition on the encrypted network

\footnotetext{
${ }^{17}$ Sometimes non-domain parts of e-mail addresses are obfuscated.
}

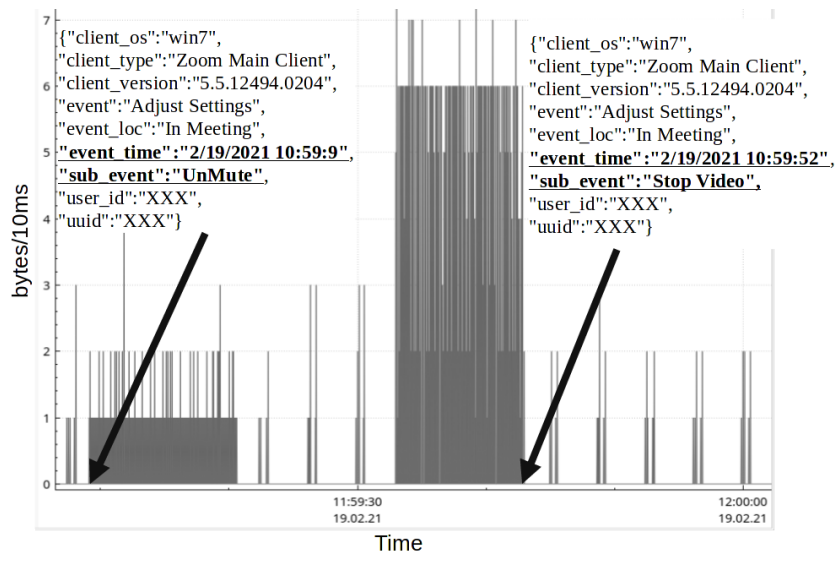

Figure 6: Events extracted from VC-related meta data and $M C S_{A U}$ correspond and can be used to confirm each other.

data streams. Confidential information about the session and the users can also be derived.

The results show that undocumented and not yet analyzed techniques of $V C S_{A}$ lead to the possibilities of separating the multimedia content streams to identify users and to derive information about sessions and user behavior.

The accessibility of this information depends on the location of the observer (which might be considered as a potential attacker) within the generalized landscape of communication (see figure 2). During our investigation, the observer is assumed to be located close to one of the clients $\left(A P_{1}\right.$ to $\left.A P_{N}\right)$. In this case, speaker identification is easier to perform if only one other client is present (neglecting the need to separate the audio signal from this stream from other signals which would be present in $M C S_{A D}$ if more clients where active). If the observer has access to at least one $M M S$ or $I S$, a set of the information presented in our paper (and potentially more) is obtainable.

If a malicious observer obtains access to decrypted content (e.g. by decrypting the TLS encrypted network data streams using Websockets during the use of $C L_{B R}$ ), information about other clients (including $E_{11}$ and $E_{12}$ ) is also accessible implying a potential multiparty privacy problem. This is also the case when the observer is situated on a $C L_{D E}$ and can obtain information usually not available on the remote $C L_{B R}$ including $E_{2}, E_{3}, E_{4}, E_{5}, E_{7}$ and $E_{8}$.

$V C S_{A}$ on-premise addresses the problem with access to MMS. However, this work showed that on-premise solutions only partly solve this potential information leak. All VC-related meta data, including log files, in-session information, account data and more are send to $V C S_{A}$ servers in any case and hence accessible to $V C S_{A}$ and third parties with access to the infrastructure.

The results of our investigation show that users privacy might still be at risk, even if the media content seems to be protected by E2E. Unusual and non obvious attacker scenarios [11] should be taken into account if one aims at rating the privacy of video conferencing solutions. 


\begin{tabular}{|c|c|c|c|c|c|c|c|}
\hline Event & Identification/ Differentiation & $M C S$ & Identifying Feature & Separator & Enc. & Client & E2E \\
\hline 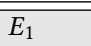 & User voice identification & $M C S_{A D}$ & Heuristics on Stream & Port & $\checkmark$ & (Any) & $\checkmark$ \\
\hline$E_{2}$ & User joins session & $M C S_{O T}, M C S_{V I}$ & Throughput Peak & Port & $\checkmark$ & $C L_{D E}$ & $\checkmark$ \\
\hline$E_{3}$ & User leaves session & $M C S_{O T}, M C S_{V I}$ & Throughput Peak & Port & $\checkmark$ & $C L_{D E}$ & $\checkmark$ \\
\hline$E_{4}$ & User muted/unmuted & $M C S_{A D}, M C S_{O T}$ & Throughput variation \& log & Port & $\checkmark$ & $C L_{D E}$ & $\checkmark$ \\
\hline$E_{5}$ & User webcam on/off & $M C S_{O T}, M C S_{V I}$ & Throughput variation \& log & Port & $\checkmark$ & $C L_{D E}$ & $\checkmark$ \\
\hline$E_{6}$ & User shares screen & $M C S_{S C}$ & New stream \& log data & Port & $\checkmark$ & All & $\checkmark$ \\
\hline$E_{7}$ & User shares video & $M C S_{V S}$ & New stream \& log data & Port & $\checkmark$ & $C L_{D E}$ & $\checkmark$ \\
\hline$E_{8}$ & Number of users & $M C S_{V I}, M C S_{A S}$ & Variation in throughput & Port & $\checkmark$ & $C L_{D E}$ & $\checkmark$ \\
\hline$E_{9}$ & Application in foreground & $M C S_{V S}$ & Sending stream stopped & Port & $\checkmark$ & All & $\checkmark$ \\
\hline$E_{10}$ & Camera Movement (from [3]) & $M C S_{V I}$ & Variation in throughput & Port & $\checkmark$ & All & $\checkmark$ \\
\hline$E_{11}$ & Users ID, E-Mail, etc. & $M C S_{O T}$ & JSON content & Websocket & $x$ & $C L_{B R}$ & $(\boldsymbol{X})$ \\
\hline$E_{12}$ & Client type, OS & $M C S_{O T}$ & JSON content & Websocket & $x$ & $C L_{B R}$ & $(\boldsymbol{X})$ \\
\hline$E_{13}$ & Text message size & $M C S_{T X}$ & Throughput (approx.) & Port \& Peak & $\checkmark$ & All & $\checkmark$ \\
\hline$E_{14}$ & User Sending file & $M C S_{F I}$ & Peak in stream & diff. $M M S$ & $\checkmark$ & $C L_{D E}$ & $\checkmark$ \\
\hline$E_{15}$ & User receiving file & $M C S_{F I}$ & Peak in stream & diff. $M M S$ & $\checkmark$ & $C L_{D E}$ & $\checkmark$ \\
\hline$E_{16}$ & File size & $M C S_{F I}$ & Throughput (exactly) & diff. $M M S$ & $\checkmark$ & $C L_{D E}$ & $\checkmark$ \\
\hline$E_{17}$ & File meta data (name \& type) & $M C S_{O T}$ & HTTP content & diff. $M M S$ & $x$ & $C L_{D E}$ & $\checkmark$ \\
\hline$E_{18}$ & User sends $V C S_{A}$ reactions & $M C S_{O T}$ & Peak in stream & Port \& Peak & $\checkmark$ & $C L_{D E}$ & $\checkmark$ \\
\hline$E_{19}$ & Any user watching & $M C S_{V I}$ & Receiving stream stopped & Port & $\checkmark$ & $C L_{D E}$ & $\checkmark$ \\
\hline$E_{20}$ & Screensharing activity & $M C S_{S C}$ & Variation in throughput & Port & $\checkmark$ & All & $\checkmark$ \\
\hline
\end{tabular}

Table 5: Comparison of all possible identifications and differentiation found by the evaluation of $V C S_{A}$ media content streams $(M C S)$. Enc.: $\checkmark$ possible on plain data, $X$ possible on decrypted content. (Any) with $E_{1}: C L_{D E}$ (only, but most likely possible with all. E2E: $\checkmark$ also possible when $E 2 E_{e n},(X)$ only visible in $C L_{B R}$ where $E 2 E_{e n}$ is not available.

\subsection{Comparison with Previous Work ([9])}

An existing dedicated forensic investigation into $\operatorname{VCS}_{A}$ (see [9]) is summarized and compared to our findings.

In [9], a forensic investigation on $V C S_{A}$ was conducted. Hard drive forensic on different client devices was chosen as the primary forensic approach. The authors also performed some memory and network forensics. For the network forensics, an HTTP proxy was employed to investigate encrypted traffic. The analyzed user functionality were not focused on the video conference sessions alone, but also included other client functionality, like the usage of chats and contact directories. Over the course of the investigations, some challenges with the protection of personal data and the anonymity of such information were discovered.

The findings of [9] and our investigation are similar with one exception: The authors state that they found no major difference between the different account types (private and educational) while various differences was identified in our paper.

Counter forensics applied by $V C S_{A}$ is reported in [9]. Our investigation also found measures which could be interpreted as such: The obfuscation of file and other content, the HTTP tunneling, the missing transparency about the employed media codecs and traffic optimization complicates every investigation about the clients behavior.

\subsection{Threats to Validity}

During the user identification test setup two different are used to avoid pattern recognition models trained to differentiate between specific connections rather than the speakers. Different text samples are used to prevent the model from learning differences in the same text samples. However, other side-effects might threaten the results: (1) Training on the different intonation based on the text instead of general speakers intonation. (2) Beneficial differences between speakers intonation. The results might not be reproducible in other participant groups where speakers have more similar intonations. (3) Multiple aspects of clean voice recording are not considered during the test setup, yet (e.g. participants were not asked to use similar distances between mouth and microphone).

As stated in [9], the (often mandatory) client updates influence the investigation. In 2020 major functionalities of the clients changed or were newly introduced. Clients received minor updates during our investigations and results might not be reproducible in future client versions.

$V C S_{A}$ offers dozens of different settings for its accounts and even more for the administrators of an educational subscription. Our settings settings during the investigation were not changed and were left in default.

\section{Summary and Outlook}

Our paper introduces a forensic framework to discern information about users and their behavior from characteristics of the encrypted network data stream of video conference sessions. The framework uses known concepts from forensics; namely the six investigation steps and the nine data types. From these concepts a generalized network landscape representing the typical setup of video conferencing solutions is derived. The six investigation steps are adapted to match network data streams typically seen in video conference sessions. The introduced framework can be applied to other video conferencing solutions with minor alterations (other approaches might be required to break the encryption, other models need to be generated for speaker identification, etc.).

To show the framework's feasibility for investigations of video conferences it is applied to $V C S_{A}$. The iterative approach of the 


\begin{tabular}{|c|c|c|}
\hline Property & Our investigation & Previous Work [9] \\
\hline Approach & $\begin{array}{l}\text { Generic framework for } \\
\text { media stream forensics in } \\
\text { video conferences, tests } \\
\text { with } V C S_{A}\end{array}$ & Dedicated $V C S_{A}$ forensics \\
\hline $\begin{array}{l}\text { Forensic } \\
\text { Types }\end{array}$ & $\begin{array}{l}\text { Network forensics, media } \\
\text { stream forensics }\end{array}$ & $\begin{array}{l}\text { Hard-Drive forensics, } \\
\text { (memory forensics), (net- } \\
\text { work forensics) }\end{array}$ \\
\hline $\begin{array}{l}\text { Account } \\
\text { Types }\end{array}$ & $\begin{array}{l}\text { Private, Educational, On- } \\
\text { Premise }\end{array}$ & Private, Educational \\
\hline Clients & $\begin{array}{l}\text { Browser, Windows 10, } \\
\text { Linux, Android }\end{array}$ & $\begin{array}{l}\text { Windows } 10 \text {, macOS, iOS, } \\
\text { Android }\end{array}$ \\
\hline $\begin{array}{l}\text { User Fea- } \\
\text { tures }\end{array}$ & In-Session & $\begin{array}{l}\text { In-Session, other client } \\
\text { functionalities }\end{array}$ \\
\hline
\end{tabular}

Table 6: Forensic results comparison in both papers.

framework helps to uncover undocumented and adaptive communication behavior of $V C S_{A}$ : clients and servers are able to switch protocols (UDP to TCP, WebRTC to Websocket, TCP and UDP to TCP HTTP-Tunnels). By modifying investigative configuration parameters (see section 4.2) the separation of different multimedia content streams and VC-related meta data is possible. These contain 20 possible means of user identifications and differentiation (see table ).

An evaluation to show the possibility to identify users based solely on the characteristics of encrypted audio streams is conducted with eight participants. This also has some implications for multi-party privacy - additional speakers in the background of a given participant might be identified using this method.

The findings show risks for users privacy. E2E encryption does not protect the large amount of plain data nor prevent identification and differentiation of users and their behavior based on encrypted content. Using $V C S_{A}$ on-premise addresses this with access to $M M S$. However, all VC-related meta data, including log files, in-session information, account data and more are always sent to the $I S$ under control of $V C S_{A}$. The test results show that users privacy is still at risk regarding first party access and third party observers (third party views), even if the MCS seems to be protected by E2E.

The application of advanced proxy techniques to reveal content of data streams to $V C S_{A}$ server that were not investigated in this work should follow.

Protocols and protocol switching mechanisms used by the provider are potential targets for information hiding - especially switching between media with different characteristics either allowing or preventing some types of detection mechanisms for malicious communication including hidden communication.

\section{Acknowledgments}

We would like to thank Felix Freiling for helpful discussions and support regarding the evaluation infrastructure.
We would further like to thank our students who either contributed ideas for additional use-cases (Yilin Chen) or supported us by serving as participants for the speaker identification (Christina Eckel, Jana Eisoldt, Johannes Behrens and Christopher Klug). The research conducted within this paper was partly funded by the European Union Project "CyberSec LSA OVGU-AMSL". In particular, the work of the media stream analysis and stream characteristics between different client setups was partly motivated and funded by the German Federal Ministry for Economic Affairs and Energy in the project STEALTH (Grant No.: 1501589A) to determine potential cover media characteristics for information hiding and the impact for its prevention.

\section{References}

[1] Robert Altschaffel. 2020. Computer forensics in cyber-physical systems : applying existing forensic knowledge and procedures from classical IT to automation and automotive. Ph.D. Dissertation. Otto von Guericke University Magdeburg.

[2] Robert Altschaffel, Robert Clausing, Christian Krätzer, Stefan Kiltz, and Jana Dittmann. 2013. Statistical pattern recognition based content analysis on encrypted network - traffic for the teamviewer application. In Seventh International Conference on IT Security Incident Management and IT Forensics (IMF 2013), Holger Morgenstern (Ed.). Springer Berlin Heidelberg, 113-121.

[3] Robert Altschaffel, Jonas Hielscher, Christian Krätzer, Kevin Lamshöft, and J. Dittmann. 2020. Forensic Behavior Analysis in Video Conferencing Based on the Metadata of Encrypted Audio and Video Streams - Considerations and Possibilities. In SECURWARE 2020: The Fourteenth International Conference on Emerging Security Information, Systems and Technologies (Valencia, Spain).

[4] Zoom Video Communications Inc. 2021. Loesungen fuer Videokonrerenzraumsysteme - Zoom. (01 2021). https://zoom.us/de-de/zoomrooms/software.html, accessed 2021/01/20.

[5] Dima Kagan, Galit Fuhrmann Alpert, and Michael Fire. 2020. Zooming Into Video Conferencing Privacy and Security Threats. arXiv:2007.01059 [cs.CR]

[6] F Karpisek, Ibrahim Baggili, and Frank Breitinger. 2015. WhatsApp network forensics: Decrypting and understanding the WhatsApp call signaling messages. Digital Investigation 15 (10 2015).

[7] Stefan Kiltz, Jana Dittmann, and Claus Vielhauer. 2015. Supporting Forensic Design - A Course Profile to Teach Forensics. In IMF '15: Proceedings of the 2015 Ninth International Conference on IT Security Incident Management \& IT Forensics (imf 2015). IEEE.

[8] Chen Ling, Utkucan Balci, J. Blackburn, and G. Stringhini. 2021. A First Look at Zoombombing. 2021 IEEE Symposium on Security and Privacy abs/2009.03822 (2021).

[9] Andrew Mahr, Meghan Cichon, Sophia Mateo, Cinthya Grajeda, and Ibrahim Baggili. 2021. Zooming into the pandemic! A forensic analysis of the Zoom Application. Forensic Science International: Digital Investigation 36 (2021), 301107.

[10] G. Palmer. 2001. A Road Map for Digital Forensic Research. Technical Report. DFRWS.

[11] Mohd Sabra, A. Maiti, and M. Jadliwala. 2020. Zoom on the Keystrokes: Exploiting Video Calls for Keystroke Inference Attacks. ArXiv abs/2010.12078 (2020).

[12] Roei Schuster, Vitaly Shmatikov, and Eran Tromer. 2017. Beauty and the Burst: Remote Identification of Encrypted Video Streams. In Proceedings of the 26th USENIX Conference on Security Symposium (Vancouver, BC, Canada) (SEC'17). USENIX Association, USA, 1357-1374.

[13] Paul Wagenseil. 2020. Zoom security issues: Here's everything that's gone wrong (so far). Toms guide (2020).

[14] Paul Wagenseil. 2021. Zoom security issues: Here's everything that's gone wrong (so far). (02 2021). https://www.tomsguide.com/news/zoom-security-privacywoes, accessed 2021/02/22.

[15] Chenggang Wang, Sean Kennedy, Haipeng Li, King Hudson, Gowtham Atluri, Xuetao Wei, Wenhai Sun, and Boyang Wang. 2020. Fingerprinting encrypted voice traffic on smart speakers with deep learning. 254-265.

[16] Nicholas Whiskerd, Nicklas Körtge, Kris Jürgens, Kevin Lamshöft, Salatiel Gomez, Claus Vielhauer, Jana Dittmann, and Mario Hildebrandt. 2020. Keystroke biometrics in the encrypted domain: a first study on search suggestion functions of web search engines. EURASIP fournal on Information Security 2020 (02 2020).

[17] Charles Wright, Lucas Ballard, Scott Coull, Fabian Monrose, and Gerald Masson. 2008. Spot Me if You Can: Uncovering Spoken Phrases in Encrypted VoIP Conversations. 35-49. 